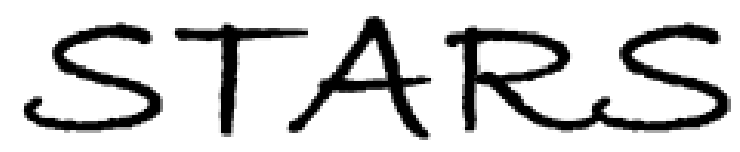

University of Central Florida

STARS

$1-1-2013$

\title{
Fidelity of the surface code in the presence of a bosonic bath
}

\author{
P. Jouzdani \\ University of Central Florida \\ E. Novais \\ E. R. Mucciolo \\ University of Central Florida
}

Find similar works at: https://stars.library.ucf.edu/facultybib2010 University of Central Florida Libraries http://library.ucf.edu

This Article is brought to you for free and open access by the Faculty Bibliography at STARS. It has been accepted for inclusion in Faculty Bibliography 2010s by an authorized administrator of STARS. For more information, please contactSTARS@ucf.edu.

\section{Recommended Citation}

Jouzdani, P.; Novais, E.; and Mucciolo, E. R., "Fidelity of the surface code in the presence of a bosonic bath" (2013). Faculty Bibliography 2010s. 4169.

https://stars.library.ucf.edu/facultybib2010/4169

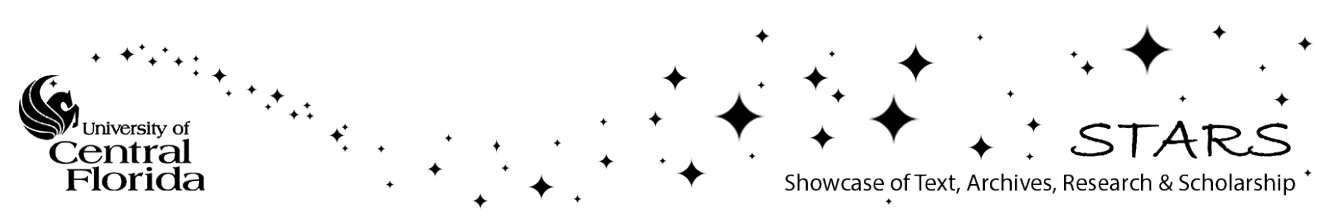




\title{
Fidelity of the surface code in the presence of a bosonic bath
}

\author{
P. Jouzdani, ${ }^{1}$ E. Novais, ${ }^{2}$ and E. R. Mucciolo ${ }^{1}$ \\ ${ }^{1}$ Department of Physics, University of Central Florida, Orlando, Florida 32816-2385, USA \\ ${ }^{2}$ Centro de Ciências Naturais e Humanas, Universidade Federal do ABC, Santo André, SP, Brazil
}

(Received 9 April 2013; published 31 July 2013)

\begin{abstract}
We study the resilience of the surface code to decoherence caused by the presence of a bosonic bath. This approach allows us to go beyond the standard stochastic error model commonly used to quantify decoherence and error threshold probabilities in this system. The full quantum mechanical system-bath dynamics is computed exactly over one quantum error correction cycle. Since all physical qubits interact with the bath, space-time correlations between errors are taken into account. We compute the fidelity of the surface code as a function of the quantum error correction time. The calculation allows us to map the problem onto an Ising-like statistical spin model with two-body interactions and a fictitious temperature which is related to the inverse bath coupling constant. The model departs from the usual Ising model in the sense that interactions can be long ranged and can involve complex exchange couplings; in addition, the number of allowed configurations is restricted by the syndrome extraction. Using analytical estimates and numerical calculations, we argue that, in the limit of an infinite number of physical qubits, the spin model sustains a phase transition which can be associated to the existence of an error threshold in the surface code. An estimate of the transition point is given for the case of nearest-neighbor interactions.
\end{abstract}

DOI: 10.1103/PhysRevA.88.012336

PACS number(s): 03.67.Lx, 03.67.Pp

\section{INTRODUCTION}

Recent progress in implementing controllable multiqubit systems in the laboratory has sparked renewed interest in topological quantum computing schemes. Particular attention has been devoted to the surface code [1-3], which is a planar version of Kitaev's toric code [4]. From a physical implementation viewpoint, the surface code has two important advantages in comparison to other schemes: (i) all gates are local, and (ii) simulations indicate that the topological protection yields very high tolerance for errors. The latter is based entirely on stochastic models for errors. These models point to error threshold probabilities per single qubit operation or cycle ranging from $1 \%[5,6]$, when only nearest-neighbor interactions and no perfect gates are assumed, up to 19\% [7], when the ability to perfectly measure four-qubit operators is assumed. The large error threshold comes at the expense of hardware: a vast number of local operations and physical qubits is required to build a useful computing machine [8]. Yet, this tradeoff seems attractive nowadays for a number of physical realizations such as cold atoms [9], ion traps [10], Rydberg atoms [11], semiconductor systems [12], and superconducting integrated systems [13].

Most error threshold estimates so far have relied on the assumption of errors being uncorrelated in time and space. However, given the large-scale integration that will be required to implement a surface code, this assumption seems unwarrantable on physics grounds. The need to have tens of millions of physical qubits siting on a common substrate and interacting with each other and with the controlling electronics is very likely to introduce environmental modes, which will effectively couple the time evolution of the physical qubits. Under these circumstances, errors will become correlated and it is unclear whether the system will retain its high error threshold. In fact, previous studies of the impact of correlated errors on standard (nontopological) quantum computing codes have shown that error thresholds may be reduced or altogether disappear in some situations [14-19]. Investigating the effect of correlations between errors in the surface code is the main goal of this paper.

In a recent paper [20], two of us showed that the time evolution of the surface code in the presence of a common bosonic bath can be mapped onto a statistical spin model. This mapping allows for the computation of the surface code fidelity much in the same way that one computes the partition function and expectation values in a spin model. As a result, the existence of an error threshold was related to the existence of a phase transition in the statistical model. Even though the interpretation of the crossing of the error threshold as a classical phase transition is not new [3,21], our formulation takes into account the full quantum-mechanical time evolution of the qubits in the presence of a dynamical environment. In addition, rather than evaluating error probabilities, we compute directly the fidelity of the logical qubit. Our choice of environment, a collection of freely propagating massless bosonic modes, is realistic for systems where decoherence can be related to the coupling to phonons, magnons, and electromagnetic modes.

Below, we provide a detailed derivation of the evolution operator of the combined surface code-bosonic bath system. We focus our attention on a single quantum error correction cycle and assume that, after the syndrome extraction, the bath is reset to its ground state. Within this approximation, we find that the fidelity can be written as a function of the expectation value of single-qubit logical operators. The study of these expectation values can be related to the physics of an Ising-like spin model with a complex fictitious temperature. Under the assumption of noncyclic and perfect stabilizer measurements, we use both exact and mean-field finite numerical calculations to argue that the spin model sustains a thermodynamic phase transition in the limit of an infinite number of physical qubits. System with 25 and 41 qubits are studied numerically. The critical temperature of the spin model yields 
a coupling constant threshold value which is found to depend mainly on bath parameters.

The paper is organized as follows. In Sec. II we give a give a brief introduction to the essential elements of the surface code and set some of the notation used later. Section III presents a Hamiltonian formulation of the problem in terms of bosonic modes coupled to physical qubits which allows us to obtain a compact form for the evolution operator of the combined logical qubit-bath system. The evolution operator involves a bath correlation function which is explicitly evaluated for three representative situations. The effect of syndrome extraction on the evolution operator is described in Sec. IV and an expression for the fidelity in terms of expectation values involving qubit operators is derived in Sec. V. The mapping of the fidelity calculation onto a statistical model is given in Sec. VI and the connection between the fictitious critical temperature and the error threshold probability is shown in Sec. VII. In Sec. VIII we estimate the fictitious critical temperature via a lowtemperature expansion. Numerical supporting the existence of an error threshold are shown in Sec. IX, which is a very encouraging result. Conclusions and a critical discussion of the approximations involved and future directions of investigation are drawn in Sec. X. A number of appendixes with technical details of the calculations are also provided.

\section{SURFACE CODE}

Following Ref. [1], we define the surface code as a collection of $N$ spins 1/2 (physical qubit systems) located on the edges of a two-dimensional lattice with two types of boundaries, as shown in Fig. 1. The lattice comprises $n$ and $m$ qubit rows and columns, respectively. Measurements are done on two types of stabilizer operators: stars $A_{\diamond}$, which are associated to lattice vertices $(\diamond)$,

$$
A_{\diamond}=\prod_{i \in \diamond} \sigma_{i}^{x}
$$

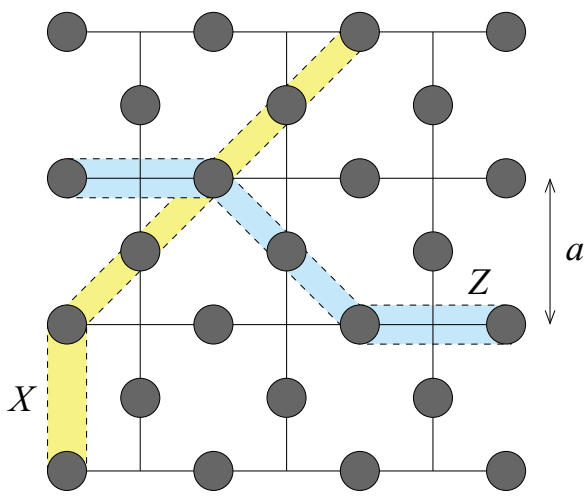

FIG. 1. (Color online) A $3 \times 3$ two-dimensional square lattice structure for a surface code. Physical qubits (circles) are located at the edges of the lattice, which has open (vertical) and closed (horizontal) boundaries. In the general case, the lattice is $n \times m$ in size, with $n m$ vertical and $(n+1)(m+1)$ horizontal edges and $N=$ physical qubits. The light colored strips show possible paths for the logical operators $\bar{Z}$ and $\bar{X}$. $a$ is the lattice constant. and plaquettes $B_{\square}$, which are associated to tiles ( $\square$ ), including the ones at the open boundaries,

$$
B_{\square}=\prod_{i \in \square} \sigma_{i}^{z} .
$$

In Eqs. (1) and (2), the Pauli spin operators $\vec{\sigma}_{i}$ act on qubits. Thus, there are $N_{\square}=(n+1) m$ plaquette operators and $N_{\diamond}=(m+1) n$ star operators. The $N$ physical qubits store one logical qubit. There are $n_{L}=2$ distinct logical operators: $\bar{X}$ and $\bar{Z}$. They are formed by a string of physical qubit operators along paths that cut through the lattice:

$$
\bar{Z}=\prod_{i \in \Gamma_{Z}} \sigma_{i}^{z}
$$

and

$$
\bar{X}=\prod_{i \in \Gamma_{X}} \sigma_{i}^{x},
$$

where $\Gamma_{Z}$ runs between qubits at opposite open boundaries (left to right), passing through vertices along the way, while $\Gamma_{X}$ runs between qubits at opposite closed boundaries (top to bottom), crossing tiles (see Fig. 1). Notice that vertices and tiles form dual lattices.

The protected code space contains two states, $|\bar{\uparrow}\rangle$ and $|\bar{\downarrow}\rangle$. Both states are eigenstates of all stabilizer operators with eigenvalue +1 . Errors can be inferred by measuring the stabilizer operators and tracking down which stars or plaquettes yielded -1 values. A decoding procedure is needed to decide which recovering operation to perform [22,23].

The code state can be generated by the action of a product involving all star operators on the $z$ ferromagnet state, namely,

$$
|\uparrow\rangle=G\left|F_{z}\right\rangle,
$$

and

$$
|\bar{\downarrow}\rangle=G \bar{X}\left|F_{z}\right\rangle
$$

where

$$
G=\frac{1}{\sqrt{2^{N_{\diamond}}}} \prod_{\diamond}\left(1+A_{\diamond}\right)
$$

and

$$
\left|F_{z}\right\rangle=\prod_{i=1}^{N}|\uparrow\rangle_{i, z}
$$

Notice that the product in Eq. (7) can be expanded as

$$
\prod_{\diamond}\left(1+A_{\diamond}\right)=1+\sum_{\diamond} A_{\diamond}+\sum_{\diamond_{1} \neq \diamond_{2}} A_{\diamond_{1}} A_{\diamond_{2}}+\cdots .
$$

The number $2^{N_{\diamond}}$ appearing in the prefactor of $G$ is the number of terms appearing in the expansion: $2^{N_{\diamond}}=\left(\begin{array}{c}N_{\diamond} \\ 0\end{array}\right)+$ $\left(\begin{array}{c}N_{\diamond} \\ 1\end{array}\right)+\cdots+\left(\begin{array}{c}N_{\diamond} \\ N_{\diamond}\end{array}\right)$.

\section{BOSONIC ENVIRONMENT}

The most general bath model would allow for both flip and phase errors to occur. However, only perturbative calculations would be possible in this general case. Since our goal is to obtain nonperturbative results, we focus our discussion on flip 
errors only (it is possible to rephrase the model to induce only the pure dephasing model by a simple change of basis). We do not explicitly consider correlated errors introduced by the hardware upon measurement, but rather errors induced by the interaction between a bath and the qubits during the time span of a QEC cycle. The Hamiltonian we consider is written as

$$
H=H_{0}+V,
$$

where $H_{0}$ is a free bosonic Hamiltonian,

$$
H_{0}=\sum_{\mathbf{k} \neq 0} \omega_{\mathbf{k}} a_{\mathbf{k}}^{\dagger} a_{\mathbf{k}}
$$

and

$$
V=\frac{\lambda}{2} \sum_{i} f\left(\mathbf{r}_{i}\right) \sigma_{i}^{x},
$$

where $\mathbf{r}_{i}$ denotes the spatial location of a qubit $i$ and $f$ is a local bosonic operator,

$$
f(\mathbf{r})=\frac{\left(v / \omega_{0}\right)^{D / 2+s}}{L^{D / 2}} \sum_{\mathbf{k} \neq 0}|\mathbf{k}|^{s}\left(e^{i \mathbf{k} \cdot \mathbf{r}} a_{\mathbf{k}}^{\dagger}+e^{-i \mathbf{k} \cdot \mathbf{r}} a_{\mathbf{k}}\right) .
$$

Here, $D$ is the bath spatial dimension, $v$ is the bosonic mode velocity, $\omega_{\mathbf{k}}=v|\mathbf{k}|$, and $\omega_{0}$ is a characteristic frequency of the bath (notice that $f$ is dimensionless since we adopt units such that $\hbar=1$ ). The creation and annihilation operators of the bosonic modes follow the standard commutation relations, namely, $\left[a_{\mathbf{k}}, a_{\mathbf{k}^{\prime}}^{\dagger}\right]=\delta_{\mathbf{k}, \mathbf{k}^{\prime}}$ and $\left[a_{\mathbf{k}}, a_{\mathbf{k}^{\prime}}\right]=\left[a_{\mathbf{k}}^{\dagger}, a_{\mathbf{k}^{\prime}}^{\dagger}\right]=0$.

The choice of $s$ depends on the physical nature of the environment and on which bosonic degree of freedom couples to the qubits. When the qubits couple directly to the bosonic displacement field, we choose $s=-1 / 2$, whereas when they couple to the bosonic current operator, we choose $s=1 / 2$ instead. Notice that these two choices allow us to write the coupling between the qubits and the bosonic environment as a simple function of the free bosonic field. Hence, they render an interaction Hamiltonian with commutators that are subluminal, namely, which are equal to zero outside the boson light cone (see below). But this is not the general rule. For instance, a model that couples the environment to two different qubit components would render an interaction Hamiltonian with nonsubluminal commutators (regardless of our choice for $s$ ).

This apparent problem comes from the fact that we usually think of errors in a dynamical sense: they happen in a point in space-time and create bosons that propagate at the speed of light. However, this is an incorrect interpretation to the equations we are about to derive. We will not be considering pulses propagating thought a medium, but rather looking at allowed normal modes of the bath and how they relate to different qubit configurations. If there is no fundamental symmetry reason for their suppression, long-wavelength modes of the bath will in general introduce superluminal effective interactions. A very simple way to highlight this fact is to rewrite the bosonic model in a coherent-state basis, $a_{\mathbf{k}}=\tilde{a}_{\mathbf{k}}+\alpha$, where $\tilde{a}_{\mathbf{k}}$ and $\tilde{a}_{\mathbf{k}}^{\dagger}$ also obey standard commutation relations and $\alpha$ in a constant. This procedure introduces an effective instantaneous interaction between the qubits as much as the Coulomb gauge introduces the instantaneous Coulomb interaction in quantum electrodynamics.
For a time interval $\Delta$, the error model comprised by Eqs. (10)-(12) leads to the following evolution operator in the interaction picture [16]:

$$
U(\Delta)=T_{t} \exp \left[-i \frac{\lambda}{2} \int_{0}^{\Delta} d t \sum_{i} f\left(\mathbf{r}_{i}, t\right) \sigma_{i}^{x}\right]
$$

Combining a Magnus expansion with the Zassenhaus formula (see Appendix A), we arrive at a remarkably simple expression for the evolution operator,

$$
\begin{aligned}
U(\Delta)= & \chi \exp \left[-\frac{\lambda^{2}}{2} \sum_{i \neq j} \Phi_{\mathbf{r}_{i} \mathbf{r}_{\mathbf{j}}}(\Delta) \sigma_{i}^{x} \sigma_{j}^{x}\right] \\
& : \exp \left[-\frac{i \lambda}{2} \sum_{i} F_{\mathbf{r}_{i}}(\Delta) \sigma_{i}^{x}\right]:,
\end{aligned}
$$

where

$$
\begin{gathered}
\Phi_{\mathbf{r s}}(\Delta)=\frac{1}{2}\left[\mathcal{G}_{\mathbf{r s}}^{(R)}(\Delta)+\mathcal{G}_{\mathbf{r s}}^{(I)}(\Delta)\right], \\
\chi=\exp \left[-\frac{\lambda^{2}}{4} \sum_{i} \Phi_{\mathbf{r}_{i} \mathbf{r}_{i}}(\Delta)\right],
\end{gathered}
$$

and :: stands for normal ordering. In Eqs. (15) and (16), we have introduced two bath correlation functions,

$$
\begin{aligned}
\mathcal{G}_{\mathbf{r s}}^{(I)}(\Delta)= & \frac{1}{2} \int_{0}^{\Delta} d t_{1} \int_{0}^{t_{1}} d t_{2}\left\{\left[f\left(\mathbf{r}, t_{1}\right), f\left(\mathbf{s}, t_{2}\right)\right]\right. \\
& \left.+\left[f\left(\mathbf{s}, t_{1}\right), f\left(\mathbf{r}, t_{2}\right)\right]\right\}
\end{aligned}
$$

and

$$
\mathcal{G}_{\mathbf{r s}}^{(R)}(\Delta)=\left\langle 0\left|F_{\mathbf{r}}(\Delta) F_{\mathbf{s}}(\Delta)\right| 0\right\rangle
$$

and the auxiliary bosonic field

$$
F_{\mathbf{r}}(\Delta)=\int_{0}^{\Delta} d t f(\mathbf{r}, t)
$$

(Notice that $\chi$ is a real number since $\mathcal{G}_{\mathrm{rr}}^{(I)}=0$.)

Below, we present the functional form of the correlations functions for two-dimensional bosonic baths $(D=2)$. Details of the calculations are provided in Appendixes B and C. We consider three representative values of the power $s$ which appears in the qubit-bath coupling constant dependence on the bath mode momentum [see Eq. (13)]. These values are $s=-1 / 2,0$, and $s=1 / 2$, corresponding to sub-Ohmic, Ohmic, and super-Ohmic baths, respectively. This classification is standard and follows from the bath's spectral function frequency dependence at low frequencies: sublinear (subOhmic), linear (Ohmic), and superlinear (super-Ohmic) [24].

\section{A. Sub-Ohmic bath}

For two-dimensional sub-Ohmic baths $(D=2$ and $s=-1 / 2$ ), the bath correlation function takes a simple closed 
form. The real part reads (see Appendix B 1)

$$
\begin{aligned}
\mathcal{G}_{\mathbf{r s}}^{(R)}(\Delta)= & -\frac{|\mathbf{r}-\mathbf{s}|}{\pi v \omega_{0}}+\frac{\Delta}{2 \omega_{0}} \theta(v \Delta-|\mathbf{r}-\mathbf{s}|) \\
& +\frac{\Delta}{\pi \omega_{0}} \theta(|\mathbf{r}-\mathbf{s}|-v \Delta)\left[\arcsin \left(\frac{v \Delta}{|\mathbf{r}-\mathbf{s}|}\right)\right. \\
& \left.+\sqrt{\frac{|\mathbf{r}-\mathbf{s}|^{2}}{(v \Delta)^{2}}-1}\right],
\end{aligned}
$$

where $\theta(x)$ denotes the Heaviside step function. The imaginary part reads (see Appendix C 1)

$$
\begin{aligned}
\mathcal{G}_{\mathbf{r s}}^{(I)}(\Delta)= & \frac{-i \Delta}{\pi \omega_{0}} \theta(v \Delta-|\mathbf{r}-\mathbf{s}|) \\
& \times\left\{\ln \left[\sqrt{\frac{(v \Delta)^{2}}{|\mathbf{r}-\mathbf{s}|^{2}}-1}+\frac{v \Delta}{|\mathbf{r}-\mathbf{s}|}\right]\right. \\
& \left.-\sqrt{1-\frac{|\mathbf{r}-\mathbf{s}|^{2}}{(v \Delta)^{2}}}\right\} .
\end{aligned}
$$

\section{B. Ohmic bath}

Choosing $D=2$ and $s=0$, the real and imaginary parts of the bath correlation function take the forms [25] (see Appendixes B 2 and C 2)

$$
\mathcal{G}_{\mathbf{r s}}^{(R)}(\Delta)=\frac{1}{\pi \omega_{0}^{2}} \operatorname{arcosh}\left(\frac{v \Delta}{|\mathbf{r}-\mathbf{s}|}\right) \theta(v \Delta-|\mathbf{r}-\mathbf{s}|),
$$

and

$$
\begin{aligned}
\mathcal{G}_{\mathbf{r s}}^{(I)}(\Delta)= & \frac{i}{\pi \omega_{0}^{2}}\left[\frac{\pi}{2} \theta(v \Delta-|\mathbf{r}-\mathbf{s}|)\right. \\
& \left.+\arcsin \left(\frac{v \Delta}{|\mathbf{r}-\mathbf{s}|}\right) \theta(|\mathbf{r}-\mathbf{s}|-v \Delta)\right] .
\end{aligned}
$$

Notice that the real part of the correlation function vanishes for distances larger than $v \Delta$. For this bath as well as others, the number of qubits within the spatial range of the correlation function is determined by the ratio $v \Delta / a$, where $a$ is the surface code lattice constant.

\section{Super-Ohmic bath}

Choosing $D=2$ and $s=1 / 2$, we find for the real part (see Appendix B 3)

$$
\mathcal{G}_{\mathbf{r s}}^{(R)}(\Delta)=\frac{v}{\pi \omega_{0}^{3}}\left[\frac{1}{|\mathbf{r}-\mathbf{s}|}-\frac{\theta(|\mathbf{r}-\mathbf{s}|-v \Delta)}{\sqrt{|\mathbf{r}-\mathbf{s}|^{2}-(v \Delta)^{2}}}\right] .
$$

For the imaginary part we find (see Appendix C 3)

$$
\mathcal{G}_{\mathbf{r s}}^{(I)}(\Delta)=\frac{i v}{\pi \omega_{0}^{3}} \frac{\theta(v \Delta-|\mathbf{r}-\mathbf{s}|)}{\sqrt{(v \Delta)^{2}-|\mathbf{r}-\mathbf{s}|^{2}}} .
$$

A schematic representation of the spatial dependence of these correlations functions is shown in Fig. 2.

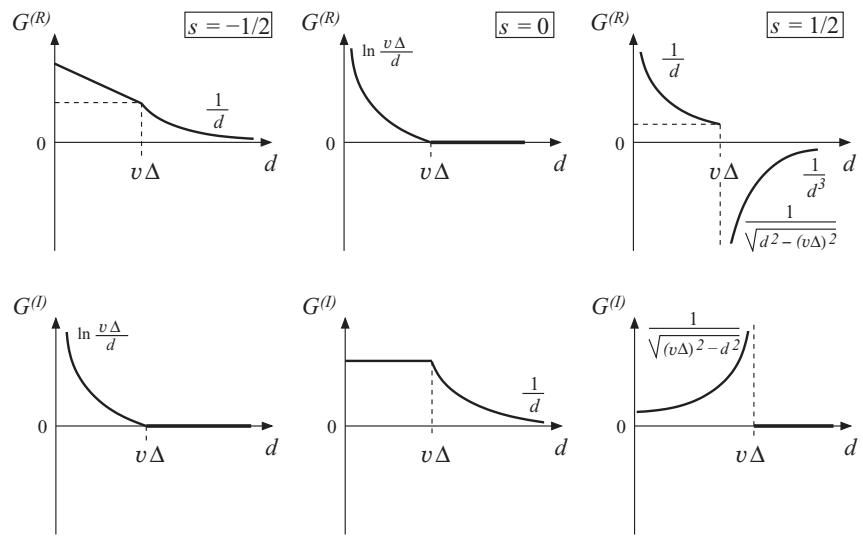

FIG. 2. Schematic representation of the spatial dependence of the correlation functions $\mathcal{G}_{\mathrm{rs}}^{(R)}(\Delta)$ and $\mathcal{G}_{\mathrm{rs}}^{(I)}(\Delta)$ for $s=-1 / 2,0$, and $1 / 2$. We use $d=|\mathbf{r}-\mathbf{s}|$.

\section{SYNDROME EXTRACTION}

Let us assume that the system is prepared initially in the logical state $|\bar{\uparrow}\rangle$ and the boson field initial state is the vacuum,

$$
\left|\Psi_{0}\right\rangle=\left(G\left|F_{z}\right\rangle\right) \otimes|0\rangle_{b}
$$

We then let the system evolve under the interaction Hamiltonian until a time $\Delta$, when an error correction protocol is performed flawlessly. The syndrome extraction operator is equivalent to the application of the projector,

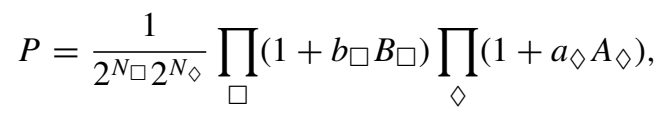

where $a_{\square}= \pm 1$ and $b_{\diamond}= \pm 1$ are the syndromes for each star and plaquette operator, respectively. Since we are assuming only bit-flip errors, the projection over stars is just the identity for $a_{\diamond}=1$, namely,

$$
P=\frac{1}{2^{N_{\square}}} \prod_{\square}\left(1+b_{\square} B_{\square}\right) .
$$

Using that $A_{\diamond} G=G$ and $[\bar{X}, G]=0$, we can rewrite the projector in a slightly different form,

$$
P=R|\uparrow\rangle\langle\bar{\uparrow}|R+R| \bar{\downarrow}\rangle\langle\bar{\downarrow}| R,
$$

where $R$ is the recovery operation chosen to be performed.

In principle, we should consider all possible syndrome outcomes. However, it is useful to look at the most benign evolution and assume $b_{\square}=1$. Such an evolution corresponds to the system remaining in the vacuum of the gauge fields after a time $\Delta$. This nonerror syndrome provides an upper bound to the available computational time. In addition, it simplifies the calculations by removing from consideration a recovery operation that tries to steer the system back to the computational basis $[22,23,26]$. Thus, for this particular case,

$$
P=|\bar{\uparrow}\rangle\langle\bar{\uparrow}|+| \bar{\downarrow}\rangle\langle\bar{\downarrow}| \text {. }
$$

The environment state is unaffected by the error correction protocol. If no extra step is taken to dissipate excitations that pile up over time, the environment will keep a memory of events that happened during the QEC period. Keeping track of such excitations between QEC cycles in a fidelity calculation 
is a difficult task even for simple, nontopological logical qubit systems $[15,16,18]$. For topological qubits, the task is considerably harder due to the exponential number of terms that enter in the composition of the computational states.

Thus, in order to make the formulation amenable to an analytical calculation, we consider an extra step to the QEC protocol. In addition to projecting the quantum computer wave function back to the logical Hilbert space, we assume that at the end of the QEC step the environment is reset to its ground state. This is equivalent to imposing at the end of the QEC step the projector $\lim _{T_{\text {bath }} \rightarrow 0} e^{-H_{0} / k_{B} T_{\text {bath }}}$, for some environment temperature $T_{\text {bath }}$ defined with respect to some even larger reservoir.

A consequence of this extra QEC hypothesis is that we exclude from the calculation any spatial correlation between QEC periods, as well as memory and spatial correlations between the time evolution of bras and kets. This new projector operator can be conveniently written as

$$
P^{\prime}=\left|\Psi_{0}\right\rangle\left\langle\Psi_{0}|+\bar{X}| \Psi_{0}\right\rangle\left\langle\Psi_{0}\right| \bar{X}
$$

After the projection, the wave function must be normalized again. For this purpose, consider the normalization factor

$$
\left\langle\Psi_{0}\left|U^{\dagger}(\Delta) P^{\prime} U(\Delta)\right| \Psi_{0}\right\rangle=|\mathcal{A}|^{2}+|\mathcal{B}|^{2},
$$

where

$$
\mathcal{A}=\left\langle\Psi_{0}|U(\Delta)| \Psi_{0}\right\rangle
$$

and

$$
\mathcal{B}=\left\langle\Psi_{0}|\bar{X} U(\Delta)| \Psi_{0}\right\rangle .
$$

Below, we use the expectation values $\mathcal{A}$ and $\mathcal{B}$ to compute the surface code fidelity after one QEC cycle.

\section{FIDELITY}

The fidelity of the surface code after one QEC cycle can be defined as

$$
F \equiv\left|\left\langle\Psi_{\mathrm{QEC}} \mid \Psi_{0}\right\rangle\right|
$$

where $\left|\Psi_{0}\right\rangle$ is the initial state of the qubit system and the bath and

$$
\left|\Psi_{\mathrm{QEC}}\right\rangle=P^{\prime} U(\Delta)\left|\Psi_{0}\right\rangle
$$

The expectation values $\mathcal{A}$ and $\mathcal{B}$ now come in handy since they allow one to obtain a simple expression for the fidelity,

$$
\begin{aligned}
F & =\left[\frac{\left\langle\Psi_{0}\left|P^{\prime} U(\Delta)\right| \Psi_{0}\right\rangle\left\langle\Psi_{0}\left|U^{\dagger}(\Delta) P^{\prime}\right| \Psi_{0}\right\rangle}{\left\langle\Psi_{0}\left|U^{\dagger}(\Delta) P^{\prime} U(\Delta)\right| \Psi_{0}\right\rangle}\right]^{1 / 2} \\
& =\left[\frac{\left\langle\Psi_{0}|U(\Delta)| \Psi_{0}\right\rangle\left\langle\Psi_{0}\left|U^{\dagger}(\Delta)\right| \Psi_{0}\right\rangle}{|\mathcal{A}|^{2}+|\mathcal{B}|^{2}}\right]^{1 / 2} \\
& =\frac{|\mathcal{A}|}{\sqrt{|\mathcal{A}|^{2}+|\mathcal{B}|^{2}}} \\
& =\frac{1}{\sqrt{1+\frac{|\mathcal{B}|^{2}}{|\mathcal{A}|^{2}}}}
\end{aligned}
$$

where we used that $P^{\prime}\left|\Psi_{0}\right\rangle=\left|\Psi_{0}\right\rangle$. Thus, our task of determining the fidelity is reduced to evaluating the ratio $|\mathcal{B}|^{2} /|\mathcal{A}|^{2}$.

\section{MAPPING ONTO A STATISTICAL MODEL}

Let us first consider the cases where the bath correlation function $\Phi_{\mathrm{rs}}(\Delta)$ has both real and imaginary parts finite, namely, $0<D+2 s<3$. The insertion of the evolution operator given by Eq. (15) into Eqs. (34) and (35) results in very compact expressions for $\mathcal{A}$ and $\mathcal{B}$ since these expectation values are taken on the bosonic vacuum. After a short manipulation, we arrive at

$$
\mathcal{A}=\chi\left\langle F_{z}\left|e^{-\beta \mathcal{H}} G^{2}\right| F_{z}\right\rangle
$$

and

$$
\mathcal{B}=\chi\left\langle F_{z}\left|\bar{X} e^{-\beta \mathcal{H}} G^{2}\right| F_{z}\right\rangle
$$

where we introduced

$$
\mathcal{H}=\frac{\lambda^{2}}{2 \beta} \sum_{i \neq j} \Phi_{\mathbf{r}_{i} \mathbf{r}_{j}}(\Delta) \sigma_{i}^{x} \sigma_{j}^{x}
$$

and

$$
\beta=\frac{1}{2 \pi}\left(\frac{\lambda}{\omega_{0}}\right)^{2} \frac{1}{\left(\omega_{0} \Delta\right)^{D+2 s-2}} .
$$

Clearly, Eq. (41) can be interpreted as an effective interaction between qubits intermediated by the environmental bosons. The connection to a statistical model becomes more apparent when we consider that the ferromagnetic state along the $z$ direction can be expanded in the $x$ basis, namely,

$$
\left|F_{z}\right\rangle=\prod_{i=1}^{N}\left(\frac{|\uparrow\rangle_{i, x}+|\downarrow\rangle_{i, x}}{\sqrt{2}}\right) .
$$

Inserting this expression into Eqs. (39) and (40), we arrive at

$$
\mathcal{A}=\frac{\chi}{2^{N}} \sum_{S} e^{-\beta E_{S}}\left\langle S\left|G^{2}\right| S\right\rangle
$$

and

$$
\mathcal{B}=\frac{\chi}{2^{N}} \sum_{S} e^{-\beta E_{S}}\left\langle S\left|\bar{X} G^{2}\right| S\right\rangle,
$$

where $S$ stands for the eigenstates of the operator $\prod_{i=1}^{N} \sigma_{i}^{x}$ and

$$
\beta E_{S}=\langle S|\beta \mathcal{H}| S\rangle \text {. }
$$

Notice that the expectation values in Eqs. (44) and (45) vanish for those states $|S\rangle$ where at least one start operator has a -1 eigenvalue. Therefore, those equations can be rewritten as

$$
\mathcal{A}=\frac{\chi}{2^{N}} \sum_{S^{\prime}} e^{-\beta E_{S^{\prime}}}
$$

and

$$
\mathcal{B}=\frac{\chi}{2^{N}} \sum_{S^{\prime}} e^{-\beta E_{S^{\prime}}}\left\langle S^{\prime}|\bar{X}| S^{\prime}\right\rangle,
$$

where the sums are over the subset of states $S^{\prime}$ where all star operators take positive values:

$$
\left\langle S^{\prime}\left|A_{\diamond}\right| S^{\prime}\right\rangle=+1
$$

It is clear now that $\mathcal{A}$ is proportional to the partition function of a classical statistical spin model with a restricted configuration space. Then, $\mathcal{C}$ is equal to the expectation value of the operator $\bar{X}$ in this model. 
The statistical model defined by Eqs. (41) and (46)-(48) is nontrivial in a number of ways. First, the interaction term (41) is not purely real. Second, the interaction range is not necessarily restricted to nearest neighbors. Third, the constraint imposed by Eq. (49) severely reduces the size of the configuration space.

Since $\left\langle S^{\prime}|\bar{X}| S^{\prime}\right\rangle$ can only take the values \pm 1 , one can rewrite Eqs. (47) and (48) as sums over "energy" eigenvalues, namely,

$$
\mathcal{A}=\frac{\chi}{2^{N}} \sum_{E^{\prime}}\left[g^{+}\left(E^{\prime}\right)+g^{-}\left(E^{\prime}\right)\right] e^{-\beta E^{\prime}}
$$

and

$$
\mathcal{B}=\frac{\chi}{2^{N}} \sum_{E^{\prime}}\left[g^{+}\left(E^{\prime}\right)-g^{-}\left(E^{\prime}\right)\right] e^{-\beta E^{\prime}},
$$

where $g^{ \pm}\left(E^{\prime}\right)$ are the number of qubit configurations with energy $E^{\prime}$ and $\langle\bar{X}\rangle= \pm 1$. The prime indicates that only configurations where all star operators have +1 expectation value are considered, Eq. (47).

When the sums in Eqs. (50) and (51) are not restricted by Eq. (47), the time-reversal symmetry of the Hamiltonian implies $g^{+}(E)=g^{-}(E)$ for logical operators $\bar{X}$ containing an odd number of $\sigma_{i}^{x}$ qubit operators. Therefore, in this case, $\mathcal{B}=0$. For $\bar{X}$ containing an even number of $\sigma_{i}^{x}$ operators, for each "energy" eigenvalue $E,\langle\bar{X}\rangle$ is either +1 [and $g_{-}(E)=0$ ] or -1 [and $g_{+}(E)=0$ ], but the value of $\mathcal{B}$ cannot be predicted.

In the case of a restricted sum, it is straightforward to see that the separation of configurations in time-reversal symmetry classes is not useful. Consider that at the vertical boundaries one can form operators $A_{\diamond}$ with three qubits. In this case, even if a certain configuration $\left|S^{\prime}\right\rangle$ satisfies Eq. (47), its time-reversal partner will not and therefore will not be included in Eqs. (50) and (51). Thus, the restriction is equivalent to projecting out time-reversal partner of $\left|S^{\prime}\right\rangle$.

As explained in Ref. [20], one useful way to understand this point is to break up the states $\left\{\left|S^{\prime}\right\rangle\right\}$ into two groups, $\left\{\left|S_{+}^{\prime}\right\rangle\right\}$ and $\left\{\left|S_{-}^{\prime}\right\rangle\right\}$, where

$$
\left|S_{+}^{\prime}\right\rangle=\prod_{j} B_{\square}\left|F_{x}\right\rangle
$$

and

$$
\left|S_{-}^{\prime}\right\rangle=\bar{Z}_{\Gamma}\left|S_{+}^{\prime}\right\rangle
$$

Here, $\prod_{j} B_{\square}$ is a product of all plaquette operators that do not touch the logical error $\bar{Z}_{\Gamma}$ for a given path $\Gamma$. It is then possible to show that this separation leads to the appearance of an effective local magnetic field that acts only on the qubits along the path $\Gamma$. This local magnetic field leads to the time-reversal symmetry breaking in the computation of the expectation values in Eqs. (50) and (51).

\section{A. Effective interaction and fictitious temperature}

The parameter $\beta$ plays the role of inverse temperature in the statistical model. From Eq. (42), we see that $\beta$ is proportional to $\lambda^{2}$, thus serving as a measure of the strength of the coupling between the qubits and the environment. The effective exchange interaction amplitude $J_{i j}$ depends on the bath characteristics (e.g., spatial dimension and spectral density), on the QEC cycle duration $\Delta$, and on the ratio $a /(\Delta v)$.

For instance, consider the Ohmic bath, where

$$
\beta=\frac{1}{2 \pi}\left(\frac{\lambda}{\omega_{0}}\right)^{2} .
$$

Using Eqs. (23) and (24), the effective Hamiltonian of the statistical model can be written as

$$
\mathcal{H}=\sum_{i \neq j} J_{i j} \sigma_{i}^{x} \sigma_{j}^{x},
$$

with

$$
J_{i j}=\frac{1}{2} \times \begin{cases}\operatorname{arcosh}\left(\frac{v \Delta}{\mid \mathbf{r}_{i}-\mathbf{r}_{j}}\right)+\frac{i \pi}{2}, & \frac{\left|\mathbf{r}_{i}-\mathbf{r}_{j}\right|}{v \Delta}<1, \\ i \arcsin \left(\frac{v \Delta}{\left|\mathbf{r}_{i}-\mathbf{r}_{j}\right|}\right), & \frac{\left|\mathbf{r}_{i}-\mathbf{r}_{j}\right|}{v \Delta}>1 .\end{cases}
$$

The real part of $J_{i j}$ is nonzero only between qubits within the light cone of the bosonic modes. The imaginary part is present for any pairs of qubits, but decays rapidly (approximately with the cube of the inverse distance) when qubits are outside the light cone. For a lattice of size $L$, an extreme limit occurs when $v \Delta \sim L$, in which case all qubits are correlated. In the opposite limit, when the QEC cycle period $\Delta$ is sufficiently short (or, equivalently, that the lattice constant is large enough), so that $a / \sqrt{2}<v \Delta<a$, only qubits belonging to the same plaquette are within the light cone defined by the free spatial propagation of the bosonic modes and the real part of the coefficient $J_{i j}$ vanishes beyond nearest neighbors.

\section{CONNECTING $\beta$ TO THE QUBIT ERROR PROBABILITY}

It is useful to relate the fictitious inverse temperature $\beta$ of the spin model to the probability $p$ of a qubit flipping its spin state during the QEC cycle. The latter can be defined as

$$
p=\left\langle 0\left|\otimes\left\langle\uparrow_{j}\left|U_{j}^{\dagger}(\Delta)\right| \downarrow_{j}\right\rangle\left\langle\downarrow_{j}\left|U_{j}(\Delta)\right| \uparrow_{j}\right\rangle \otimes\right| 0\right\rangle,
$$

where $\left\{\left|\uparrow_{j}\right\rangle,\left|\downarrow_{j}\right\rangle\right\}$ are states of the qubit located at $\mathbf{r}_{j},|0\rangle$ is the bath ground state, and

$$
U_{j}(\Delta)=T_{t} \exp \left[-\frac{\lambda}{2} \int_{0}^{\Delta} d t f\left(\mathbf{r}_{j}, t\right) \sigma_{j}^{x}\right]
$$

is the evolution operator of that qubit coupled to the bath when the dynamics of all other qubits is frozen. The steps in the evolution of Eq. (57) are similar to those used in the derivation of the fidelity. The details are provided in Appendix D. The result is

$$
p=\frac{1}{2}\left\{1-\exp \left[-\frac{\lambda^{2}}{4} \mathcal{G}_{\mathbf{r}_{j} \mathbf{r}_{j}}^{(R)}(\Delta)\right]\right\} .
$$

Notice that for $\lambda=0, p=0$. As the coupling between qubits and bath grows in magnitude, $p$ approaches $1 / 2$, which signals a complete randomization of the qubit state.

The functional relation between $p$ and the fictitious temperature $\beta$ can be easily established by evoking Eq. (42) and employing the explicit form of $\mathcal{G}_{\mathbf{r}_{j} \mathbf{r}_{j}}^{(R)}(\Delta)$ as given in Eq. (B1). 
One obtains

$\ln (1-2 p)=-\frac{\pi \beta(v \Delta)^{D+2 s-2}}{L^{D}} \sum_{\mathbf{k} \neq 0}|\mathbf{k}|^{2 s-2}[1-\cos (|\mathbf{k}| v \Delta)]$.

The sum over momentum diverges in the ultraviolet when $D+2 s \geqslant 2$ and the relation between the error probability and the fictitious inverse temperature becomes cutoff dependent. For instance, for $D=2$ and $s=0$ (Ohmic bath), one finds $p=$ $\frac{1}{2}\left[1-(2 v \Delta \Lambda)^{-\beta / 2}\right]$, where $\Lambda$ is the ultraviolet momentum cutoff. However, for $D=2$ and $s=-1 / 2$ (sub-Ohmic case), one finds $p=\frac{1}{2}\left(1-e^{-\pi \beta / 4}\right)$, which is cutoff independent.

\section{ESTIMATE OF $\boldsymbol{\beta}_{c}$}

We now provide an estimate of the critical inverse fictitious temperature, taking a slightly different approach from that used in Ref. [20]. Let us consider the case when the effective spin coupling in Eq. (41) is real and only nearest-neighbor interactions occur, $\Phi_{\mathbf{r}_{i} \mathbf{r}_{j}}(\Delta)=J$ for $\left|\mathbf{r}_{i}-\mathbf{r}_{j}\right| \leqslant a / \sqrt{2}$ and $\Phi_{\mathbf{r}_{i} \mathbf{r}_{j}}(\Delta)=0$ otherwise. We will carry out a low-temperature (large $\beta$ ) expansion of Eqs. (50) and (51). The key element we exploit in this expansion is the following property of the surface code: At the boundaries of the surface code, the star operators are defined by three qubits instead of four. This means that if a certain state $|S\rangle$ is included in the restricted sums defining $\mathcal{A}$ and $\mathcal{B}$, its time-reversed counterpart is included. This is because reversing all the qubit of a configuration with an eigenvalue +1 for all star operators yields a -1 eigenvalue for the star operators at the boundaries.

This property is particularly useful in the limit of $\beta \rightarrow \infty$ when the term with the minimum energy, $e^{-\beta E_{\min }^{\prime}}$, carries the leading contribution to the sums. For $\beta \rightarrow \infty$ and for $J<0$, the only state with minimum energy is a ferromagnetic state where all spins are pointing along the positive $x$ direction, $\left|F_{x}\right\rangle$. Since the the ferromagnet state with spins pointing along the negative $x$ is not part of the restricted sum, $g^{-}\left(E_{\min }^{\prime}\right)=0$. In this limit, the spin model is in the ordered phase, with $\mathcal{A}=\mathcal{B}$, resulting in $\mathcal{F}=1 / 2$ (lost fidelity).

Consider now a large but finite $\beta$. Starting from the state $\left|F_{x}\right\rangle$, the states appearing in $\mathcal{A}$ and $\mathcal{B}$ can be separated into two groups, as shown in Eqs. (52) and (53). The first group, $\left\{S_{+}^{\prime}\right\}$, corresponds to the states counted in $g^{+}\left(E^{\prime}\right)$, whereas the second group, $\left\{S_{+}^{\prime}\right\}$, is accounted for by $g^{-}\left(E^{\prime}\right)$. Therefore, $g^{-}\left(E^{\prime}\right)$ is equal to the number of states of energy $E^{\prime}$ with a logical error $\bar{Z}_{\Gamma}$ for a given path $\Gamma$. At large $\beta$, the energy cost of these states is of the order of the length of $\bar{Z}_{\Gamma}$ and they are suppressed in comparison to other states. The leading terms contributing to the sums are the minimum energy state $\left|F_{x}\right\rangle$ and the states $\left|S_{+}^{\prime}\right\rangle$ containing only small loops. However, as the system size increases the multiplicity factor $g^{+}\left(E^{\prime}\right)$ increases as well. Its value is proportional to the number of ways one can apply the $\bar{Z}$ operation, or equivalently, to the number of self-avoiding walks (SAWs) from one open boundary to its opposite. The number of SAWs with a length $l$ is related to connective constant $\mu$ of the lattice and scales as $\mu^{l}$.

If the multiplicity factor $g^{-}\left(E^{\prime}\right)$ is high enough, it can compensate the Boltzmann factor suppression. Then, as $\beta$ decreases to a certain value $\beta_{c}$, for some energy $E_{\star}^{\prime}$ a term of the type $\mu^{l_{\bar{Z}}\left(E_{\star}^{\prime}\right)} e^{-\beta_{c} E_{\star}^{\prime}}$ will appear in $\mathcal{B}$ with the same order as the leading term related to the $\left|S_{+}^{\prime}\right\rangle$ states, namely, $e^{-\beta_{c} E_{\min }^{\prime}}$. Here, $l_{\bar{Z}}$ is the length of $\bar{Z}$. This criterion provides a crude estimate for $\beta_{c}$ :

$$
e^{-\beta_{c} E_{\min }^{\prime}} \approx \mu^{l_{\bar{Z}}\left(E_{\star}^{\prime}\right)} e^{-\beta_{c} E_{\star}^{\prime}}
$$

leading to

$$
\beta_{c} \approx \frac{l_{\bar{Z}}\left(E_{\star}^{\prime}\right) \ln \mu}{E_{\star}^{\prime}-E_{\min }^{\prime}} .
$$

The difference between $E_{\star}^{\prime}$ and $E_{\min }^{\prime}$ is proportional to the length of the logical error $\bar{Z}$. Then, the denominator is of the order of $2 n l_{\bar{Z}} J$, where $n$ is the number of qubits interacting with the qubits comprising the logical error $\bar{Z}$ through the Hamiltonian in Eq. (55). A range of possible values for the connective constant of a square lattice can be found in the literature. If we adopt $\mu=2.64$ [27], set $n=4$, and insert these values into Eq. (62), we obtain $\beta_{c} J \approx 0.12$.

\section{NUMERICAL EVALUATION OF THE FIDELITY}

In light of Sec. VI, we can interpret the ratio $\mathcal{B} / \mathcal{A}$ as the expectation value of the $M_{x}=\sum_{j \in \Omega} \sigma_{j}^{x}$, namely, the $x$ magnetization of a linear set of spin- $1 / 2$ particles embedded into a spin system governed by the Hamiltonian of Eq. (55) with the restriction imposed by Eq. (47). (Here, $\Omega$ denotes the path defining the logical error $\bar{X}$.) In the absence of such a restriction, the computation of $\mathcal{B} / \mathcal{A}$ in the thermodynamic limit would follow standard procedures used in statistical mechanics. The restriction, however, makes an analytical computation rather difficult, if not impossible. Therefore, we resort to numerical calculations, both exact and approximate, to find how $\mathcal{B}$ and $\mathcal{A}$ (and thus the fidelity $\mathcal{F}$ ) behave as a function of $\beta$ and how this behavior scales with increasing system sizes.

Below, we focus on the case where the effective interaction strength $J_{i j}$ is real and only involves nearest-neighbor qubits. As mentioned earlier, this special case is of significance to experiments where $v \Delta$ is of the order of $a$. Short-range correlated errors in this case can be introduced by any measurement or operation on individual stars and plaquettes.

\section{A. Exact calculations}

For two lattice sizes, $N=25$ and 41 , we computed $\mathcal{A}$ and $\mathcal{B}$ for an $\bar{X}$ operator that ran vertically through the middle of the lattice. The computation was done by exhaustive enumeration of all orthogonal qubit configurations $|S\rangle$ that complied with the constraint $\left\langle S\left|G^{2}\right| S\right\rangle \neq 0$, namely, that produced only positive plaquette eigenvalues. We verified that the results were insensitive to the choice of operator $\bar{X}$. The resulting fidelity is shown in Fig. 3 as a function of the inverse fictitious temperature $\beta$. For small $\beta$ (equivalent to small coupling constant $\lambda$ ), the fidelity stays close to 1 after one QEC cycle. As $\beta$ increases, the fidelity decays and tends asymptotically to $1 / \sqrt{2}$, which is expected when $\mathcal{B}=0$. Another important feature is that the transition from $\mathcal{F}=1$ to $\mathcal{F}=1 / \sqrt{2}$ becomes sharper as the system size is increased. This is the expected behavior when, in the thermodynamic, 


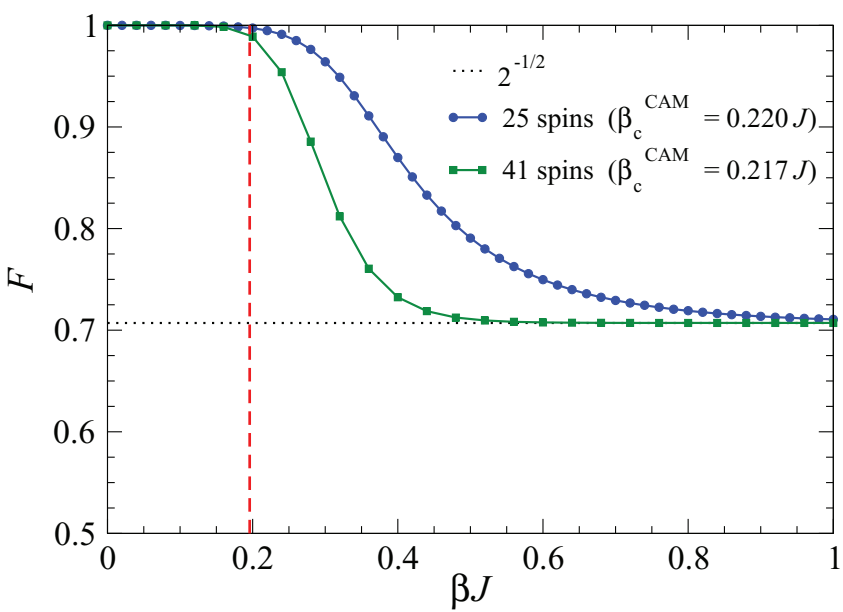

FIG. 3. (Color online) Surface code fidelity of code spaces of 25 and 41 physical qubits in contact with a bosonic bath when star operators are restricted to positive values $\left(A_{\diamond}=1\right)$.

infinite-size limit, a phase transition occurs at some critical value of $\beta$.

We have tested that this behavior is not substantially altered when the coupling constant $J$ gains a constant imaginary part. The results are shown in Fig. 4. The main effect of adding an imaginary part is to create oscillations in the decay of
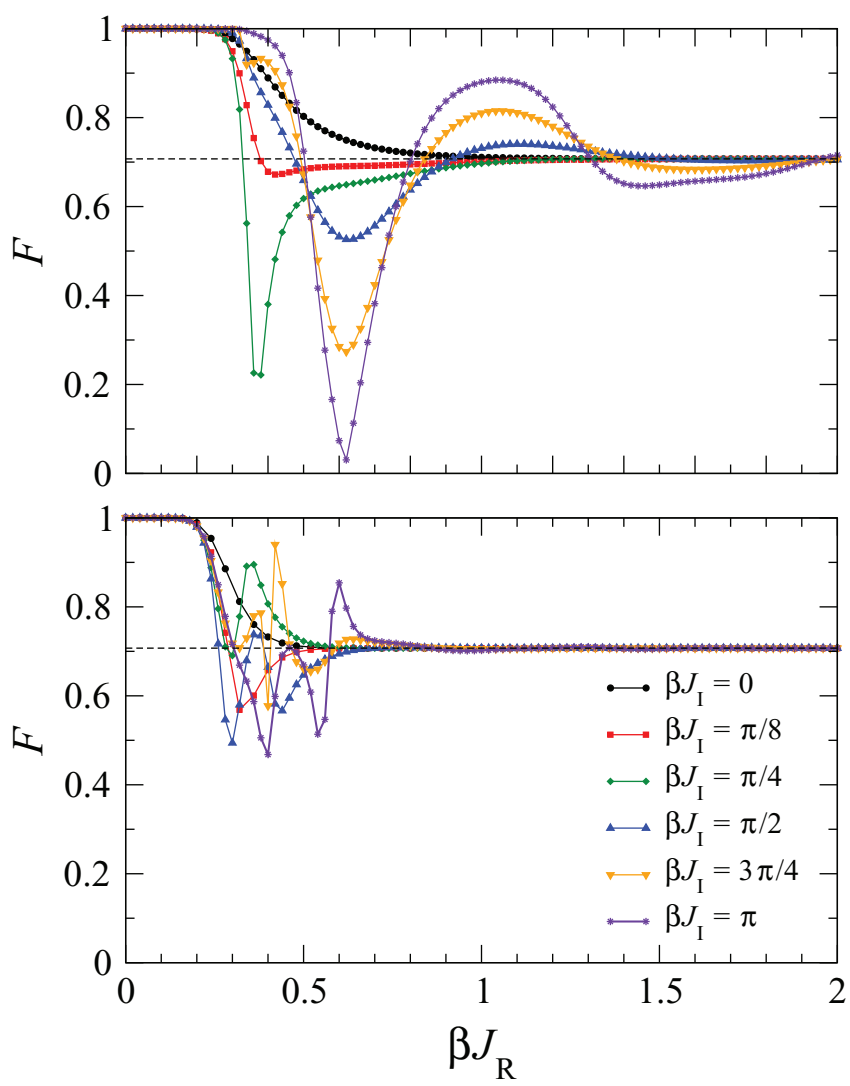

FIG. 4. (Color online) Fidelity of a code space of 25 physical qubits in contact with a bosonic bath when star operators are restricted to positive values $\left(A_{\diamond}=1\right)$ and an imaginary part is added to the coupling constant: $J=J_{R}+i J_{I}$. The data sets correspond to different values of $J_{I}$. the fidelity as a function of $\beta$. The larger the magnitude of the imaginary part in $J$, the more oscillations are observed. However, the relative amplitude of these oscillations decreases with increasing system size. In the limit of a large number of physical qubits, we expect the oscillations to be relatively small and concentrated near the critical value $\beta_{c}$.

In order to determine the critical value $\beta_{c}$, we resort to the coherent anomaly method, which has been extensively and successfully used to determine critical temperatures in interacting spin systems [28,29].

\section{B. Mean-field solution: Coherent anomaly method}

In the coherent anomaly method (CAM), a cluster of interacting spins is embedded inside a mean-field medium. Self-consistency is obtained by allowing the spins at the boundary of the cluster to experience the mean field, which is set equal to the mean value of the central spin in the cluster. This constraint provides an equation from which the critical temperature can be determined. As the cluster size is increased, the expectation is that the critical temperature obtained in this way rapidly approaches the exact value of an infinite-size system [28,29].

More precisely, let $S_{0}^{x}$ denote the central spin operator and let $\mathcal{H}$ be a Hamiltonian describing nearest-neighbor interactions inside the cluster $\mathcal{H}_{\mathrm{cl}}$ as well as the action of an effective field $\phi_{\text {eff }}$ at the boundary spins,

$$
\mathcal{H}=\mathcal{H}_{\text {cal }}+J \phi_{\text {eff }} \sum_{i \in \partial \Omega} S_{i}^{x},
$$

where $\partial \Omega$ denotes the cluster boundary. The expectation value of the central spin is given by

$$
\left\langle S_{0}^{x}\right\rangle=\frac{\operatorname{Tr}\left[S_{0}^{x} e^{-\beta \mathcal{H}}\right]}{\operatorname{Tr}\left[e^{-\beta \mathcal{H}}\right]},
$$

where the trace is carried over all allowed spin configurations. Expanding the exponentials in Eq. (63) to the lowest nontrivial order in the effective field, we find that

$$
\left\langle S_{0}^{x}\right\rangle=\left\langle S_{0}^{x}\right\rangle_{\mathrm{cl}}-\beta J \phi_{\mathrm{eff}} \sum_{i \in \partial \Omega}\left\langle S_{0}^{x} S_{i}^{i}\right\rangle_{\mathrm{cl}}
$$

where $\langle\cdots\rangle_{\mathrm{cl}}$ denotes the expectation value taken with just the Hamiltonian $\mathcal{H}_{\mathrm{cl}}$ and neglecting the boundary field. Setting $\left\langle S_{0}^{x}\right\rangle$ equal to $\phi_{\text {eff }}$, we arrive at the equation

$$
1-\beta_{c} J \sum_{i \in \partial \Omega}\left\langle S_{0}^{x} S_{i}^{x}\right\rangle_{\mathrm{cl}}=0,
$$

which can be solved numerically to yield the critical inverse temperature $\beta=\beta_{c}$ as a function of $J$. The most costly part of the procedure is the calculation of the correlation function $\left\langle S_{0}^{x} S_{i}^{x}\right\rangle_{\mathrm{cl}}$, which requires an exhaustive enumeration of all spin configurations within the cluster.

We employed this method to compute the critical value of $\beta$ for surface codes with clusters of increasing sizes and performed a finite-scaling analysis to estimate the critical value in the thermodynamic limit. The result is shown in Fig. 5. As in the case of the exact numerical calculations, we employed the constrained nearest-neighbor Ising model of Sec. VI with $J$ real and only allowed for spin configurations with positive plaquette eigenvalues. We find that $\beta_{c} J=0.193(2)$ for an 


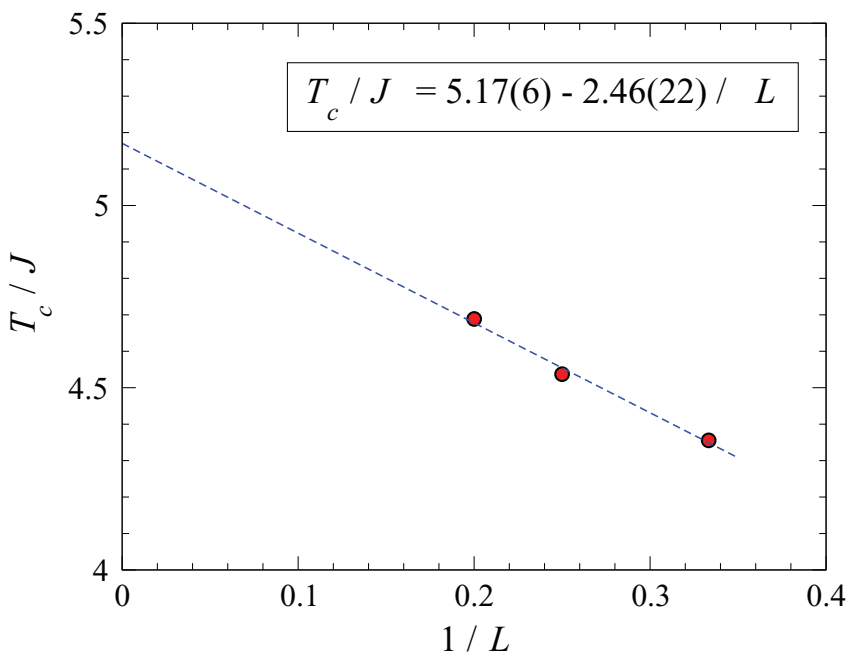

FIG. 5. (Color online) Finite-size scaling of the critical fictitious temperature $T_{c}$ obtained from cluster mean-field calculations for lattice of sizes 13, 25, and 41. A real Ising interaction of strength $J$ involving only nearest neighbors was used. The circles are the numerical data and the dashed line is a linear fit. $L$ denotes the linear size of the surface code.

infinitely large system, which is about $60 \%$ higher than the estimate presented in Sec. VIII (given the roughness of the approximations involved in the estimate, the discrepancy seems quite acceptable). The extrapolated value also matches quite closely the point where the downturn of the fidelity develops (see Figs. 3 and 4), providing additional support for the existence of a phase transition in the thermodynamic limit in the case of nearest-neighbor interactions.

\section{DISCUSSION AND CONCLUSIONS}

We have presented a fully quantum-mechanical calculation of the fidelity of the surface code in the presence of a bosonic bath. We considered the fidelity after a complete quantum error correction cycle and in the most benign case, when a nonerror syndrome occurs. An important assumption made in the calculation was the resetting of the bath to its ground state after the syndrome extraction. We then expressed the fidelity as a function of the ratio of two complex amplitudes which were formulated as expectation values of a statistical spin model with complex two-body interactions and a restricted configuration space. We presented both analytical estimates and numerical evidence that the statistical spin model sustains an ordered to disordered phase transition in the limit of an infinite number of qubits. The existence of such a phase transition can be directly related to the existence of a threshold on resilience of the surface code: provided that the bath coupling constant remains lower than a critical value, fidelity can be maintained close to unity with increasing system size. This is very good news for those interested in large-scale implementations of the surface code.

This work provides more detailed derivations and deeper analyses than Ref. [20], in additional to numerical support to the existence of an error threshold in the surface code in the presence of correlated noise. We note that our approach differs substantially from the surface code literature since we do not rely on a stochastic error model.

In order to understand the difference between a threshold due to correlations and the usual stochastic model discussion, let us consider the case of $D=2$ and $s=0$ (Ohmic bath). As shown in Sec. VII, the probability of such a bosonic bath to produce a bit flip error is $p=\frac{1}{2}\left[1-(2 v \Delta \Lambda)^{-\beta / 2}\right]$. If we take the ultraviolet cutoff to infinity, then we are bound to find $p=1 / 2$. However, in most physics systems, and condensedmatter systems in particular, the cutoff is finite. Hence, we can expand $p$ for small $\beta$ and $\Delta$ to obtain

$$
\beta \approx \frac{4 p}{\ln |2 v \Delta \Lambda|} \text {. }
$$

For the Ohmic model, we also found that the real part of the effective interaction is given by [see Eq. (56)]

$$
J_{i j} \approx \frac{1}{2} \ln \left(\frac{v \Delta}{\left|\mathbf{r}_{i}-\mathbf{r}_{j}\right|}\right)
$$

for qubits within the causality cone. In both equations, the logarithms are slow growing functions and should be regarded as producing numbers of the same order. Therefore, we can rewrite $p$ as

$$
\beta \approx \frac{2 p}{J},
$$

where we took $J \sim J_{i j} \sim(1 / 2) \ln |2 v \Delta \Lambda|$. Now, we have also found that the inverse critical temperature is [see Eq. (62)]

$$
\beta_{c} \approx \frac{\ln \mu}{2 n J} .
$$

To be resilient to correlated errors, we must require the system to be above the critical temperature, thus $\beta<\beta_{c}$. Using the equations above, we find that

$$
p<\frac{\ln \mu}{4 n} .
$$

For the least correlated case, where only nearest-neighbors effective interactions take place, we have

$$
p<\frac{\ln \mu}{16} \sim 6 \% \text {. }
$$

That is, the threshold for the surface code in the presence of an Ohmic bath is reduced to at most $p_{c} \sim 6 \%$ due to the introduction of nearest-neighbors correlated errors. If we allow for longer-range correlated errors, the threshold will steadily decline.

Within our formulation, exact analytical calculations of the threshold based on the fidelity are daunting. Thus, it is likely that quantitative results will always require numerical simulations. We are in the process of simulating statistical spin models with more general interactions than the nearestneighbor case investigated here. In addition, further investigations are necessary to relax the assumption of bath resetting and to evaluate the effects of residual qubit correlations on the fidelity over multiple cycles. Such studies are also under way.

\section{ACKNOWLEDGMENTS}

We acknowledge insightful conversations with R. Raussendorf and thank him for the hospitality at the 
University of British Columbia, Canada, where this work was initiated. E.N. and E.M. also thank L. G. Dias at the Universidade de São Paulo, Brazil, for his hospitality. E.N. was partially supported by INCT-IQ and CNPq (Brazil). This work was supported in part by the Office of Naval Research and the National Science Foundation (USA).

\section{APPENDIX A: EVOLUTION OPERATOR}

Consider Eq. (14), where the bosonic field in the interaction picture reads

$$
\begin{aligned}
f(\mathbf{r}, t)= & \frac{\left(v / \omega_{0}\right)^{D / 2+s}}{L^{D / 2}} \sum_{\mathbf{k} \neq 0}|\mathbf{k}|^{s}\left(e^{i \mathbf{k} \cdot \mathbf{r}-i|\mathbf{k}| v t} a_{\mathbf{k}}^{\dagger}\right. \\
& \left.+e^{-i \mathbf{k} \cdot \mathbf{r}+i|\mathbf{k}| v t} a_{\mathbf{k}}\right) .
\end{aligned}
$$

We can write $U(\Delta)=\exp [\Omega(\Delta)]$, where $\Omega(\Delta)$ follows the Magnun expansion

$$
\Omega(\Delta)=\Omega_{1}(\Delta)+\Omega_{2}(\Delta)+\Omega_{3}(\Delta)+\cdots
$$

with

$$
\begin{aligned}
\Omega_{1}(\Delta)= & -\frac{i \lambda}{2} \int_{0}^{\Delta} d t \sum_{i} f\left(\mathbf{r}_{i}, t\right) \sigma_{i}^{x}, \\
\Omega_{2}(\Delta)= & -\frac{1}{2 !}\left(\frac{\lambda}{2}\right)^{2} \int_{0}^{\Delta} d t_{1} \int_{0}^{t_{1}} d t_{2} \\
& \times \sum_{i, j}\left[f\left(\mathbf{r}_{i}, t_{1}\right), f\left(\mathbf{r}_{j}, t_{2}\right)\right] \sigma_{i}^{x} \sigma_{j}^{x}, \\
\Omega_{3}(\Delta)= & -\frac{i}{3 !}\left(\frac{\lambda}{2}\right)^{3} \int_{0}^{\Delta} d t_{1} \int_{0}^{t_{1}} d t_{2} \int_{0}^{t_{2}} d t_{3} \\
& \times \sum_{i, j, k}\left(\left[f\left(\mathbf{r}_{i}, t_{1}\right),\left[f\left(\mathbf{r}_{j}, t_{2}\right), f\left(\mathbf{r}_{k}, t_{3}\right)\right]\right]\right. \\
& \left.+\left[f\left(\mathbf{r}_{k}, t_{3}\right),\left[f\left(\mathbf{r}_{j}, t_{2}\right), f\left(\mathbf{r}_{i}, t_{1}\right)\right]\right]\right) \\
& \times \sigma_{i}^{x} \sigma_{j}^{x} \sigma_{k}^{x},
\end{aligned}
$$

etc. Since $\left[f\left(\mathbf{r}_{i}, t_{1}\right), f\left(\mathbf{r}_{j}, t_{2}\right)\right]$ is a $c$ number, only the first two terms in the expansion survive and we can write

$$
\begin{aligned}
U(\Delta)= & \exp \left[-i \frac{\lambda}{2} \sum_{i} F_{\mathbf{r}_{i}}(\Delta) \sigma_{i}^{x}\right] \\
& \times \exp \left[-\frac{\lambda^{2}}{8} \sum_{i, j} \mathcal{G}_{\mathbf{r}_{i} \mathbf{r}_{j}}^{(I)}(\Delta) \sigma_{i}^{x} \sigma_{j}^{x}\right] .
\end{aligned}
$$

where $\mathcal{G}_{\mathbf{r}_{i} \mathbf{r}_{j}}^{(I)}(\Delta)$ and $F_{\mathbf{r}_{i}}(\Delta)$ are defined in Eqs. (18) and (20), respectively.

It is convenient to rewrite the first exponential in Eq. (A6) as a normal ordered term. For this purpose, we note that $F_{\mathbf{r}_{i}}(\Delta)$ has the form

$$
F_{\mathbf{r}_{i}}(\Delta)=\sum_{k \neq 0}\left(g_{\mathbf{r}_{i}, \mathbf{k}}^{*} a_{\mathbf{k}}+g_{\mathbf{r}_{i}, \mathbf{k}} a_{\mathbf{k}}^{\dagger}\right),
$$

where

$$
g_{\mathbf{r}, \mathbf{k}}=-i \frac{\left(v / \omega_{0}\right)^{D / 2+s}}{v L^{D / 2}}|\mathbf{k}|^{s-1} e^{i \mathbf{k} \cdot \mathbf{r}}\left(e^{i|\mathbf{k}| v \Delta}-1\right) .
$$

Thus, we can write

$$
\exp \left[-\frac{i \lambda}{2} \sum_{i} F_{\mathbf{r}_{i}}(\Delta) \sigma_{i}^{x}\right]=\prod_{\mathbf{k} \neq 0} \exp \left(a_{\mathbf{k}}^{\dagger} v_{\mathbf{k}}-a_{\mathbf{k}} v_{\mathbf{k}}^{\dagger}\right),
$$

where

$$
v_{\mathbf{k}}=-\frac{i \lambda}{2} \sum_{i} g_{\mathbf{r}_{i}, \mathbf{k}} \sigma_{i}^{x} .
$$

Now we can use the Zassenhaus formula applied to bosonic operators,

$$
e^{\left(a_{\mathbf{k}}^{\dagger} v_{\mathbf{k}}-a_{\mathbf{k}} v_{\mathbf{k}}^{*}\right)}=e^{(1 / 2)\left[a_{\mathbf{k}}^{\dagger} v_{\mathbf{k}}, a_{\mathbf{k}} v_{\mathbf{k}}^{\dagger}\right]} e^{a_{\mathbf{k}}^{\dagger} v_{\mathbf{k}}} e^{-a_{\mathbf{k}} v_{\mathbf{k}}^{\dagger},}
$$

which results in

$$
\begin{aligned}
\exp \left[-i \lambda \sum_{i} F_{\mathbf{r}_{i}}(\Delta) \sigma_{i}^{x}\right]= & \prod_{\mathbf{k} \neq 0} e^{-(1 / 2) v_{\mathbf{k}} v_{\mathbf{k}}^{\dagger}} e^{a_{\mathbf{k}}^{\dagger} v_{\mathbf{k}}} e^{-a_{\mathbf{k}} v_{\mathbf{k}}^{\dagger}} \\
= & \prod_{\mathbf{k} \neq 0} e^{-(1 / 2) v_{\mathbf{k}} v_{\mathbf{k}}^{\dagger}}: e^{a_{\mathbf{k}}^{\dagger} v_{\mathbf{k}}-a_{\mathbf{k}} v_{\mathbf{k}}^{\dagger}}: \\
= & e^{-(1 / 2) \sum_{\mathbf{k} \neq 0} v_{\mathbf{k}} v_{\mathbf{k}}^{\dagger}} \\
& \times: \exp \left[-i \frac{\lambda}{2} \sum_{i} F_{\mathbf{r}_{i}}(\Delta) \sigma_{i}^{x}\right]:
\end{aligned}
$$

where $:(\ldots)$ : denotes normal ordering. We can rewrite the argument of the first exponential in Eq. (A12) since

$$
\begin{aligned}
\sum_{\mathbf{k} \neq 0} v_{\mathbf{k}} v_{\mathbf{k}}^{\dagger} & =-\frac{\lambda^{2}}{4} \sum_{i, j}\left(\sum_{\mathbf{k} \neq 0} g_{\mathbf{r}_{i}, \mathbf{k}} g_{\mathbf{r}_{j}, \mathbf{k}}^{*}\right) \sigma_{i}^{x} \sigma_{j}^{x} \\
& =-\frac{\lambda^{2}}{4} \sum_{i, j} \mathcal{G}_{\mathbf{r}_{i} r_{j}}^{(R)}(\Delta) \sigma_{i}^{x} \sigma_{j}^{x},
\end{aligned}
$$

where $\mathcal{G}_{\mathbf{r}_{i} \mathbf{r}_{j}}^{(R)}(\Delta)$ is defined in Eq. (19). Combining Eqs. (A6), (A12), and (A13), we arrive at Eq. (15).

\section{APPENDIX B: CORRELATOR $\mathcal{G}_{\mathrm{rs}}^{(R)}(\Delta)$}

The correlator in Eq. (19) can be evaluated in the following way. Inserting Eq. (20) into Eq. (19) and using Eq. (A1), we find

$$
\mathcal{G}_{\mathrm{rs}}^{(R)}(\Delta)=\frac{\left(v / \omega_{0}\right)^{D+2 s}}{v^{2} L^{D}} \sum_{\mathbf{k} \neq 0}|\mathbf{k}|^{2 s-2} e^{i \mathbf{k} \cdot(\mathbf{r}-\mathbf{s})}\left|e^{i|\mathbf{k}| v \Delta}-1\right|^{2} .
$$

This is integral is convergent provided that $0<D+2 s<4$. Assuming $D=2$, we can write

$$
\begin{aligned}
\mathcal{G}_{\mathbf{r s}}^{(R)}(\Delta)= & \frac{2\left(v / \omega_{0}\right)^{2+2 s}}{v^{2}} \int \frac{d^{2} k}{(2 \pi)^{2}}|\mathbf{k}|^{2 s-2} e^{i \mathbf{k} \cdot(\mathbf{r}-\mathbf{s})} \\
& \times[1-\cos (|\mathbf{k}| v \Delta)] \\
= & \frac{\left(v / \omega_{0}\right)^{2+2 s}}{\pi v^{2}} \int_{0}^{\Lambda} d k k^{2 s-1} J_{0}(k|\mathbf{r}-\mathbf{s}|) \\
& \times[1-\cos (k v \Delta)],
\end{aligned}
$$

where $J_{n}(x)$ is the $n$th Bessel function of the first kind. To proceed further, we consider three representative values 
of $s$ where the integration over momentum is convergent independent of the cutoff and we can set $\Lambda \rightarrow \infty$.

\section{Sub-Ohmic case}

For $s=-1 / 2$ we can write

$$
\mathcal{G}_{\mathrm{rs}}^{(R)}(\Delta)=\frac{1}{\pi v \omega_{0}} \int_{0}^{\infty} \frac{d k}{k^{2}} J_{0}(k|\mathbf{r}-\mathbf{s}|)[1-\cos (k v \Delta)] .
$$

Then, Eq. (21) can be obtained by using the integral [30]

$$
\begin{aligned}
& \int_{0}^{\infty} \frac{d x}{x^{2}} J_{0}(\beta x)[1-\cos (\alpha x)] \\
& \quad=-\beta+ \begin{cases}\sqrt{\beta^{2}-\alpha^{2}}+\alpha \arcsin \left(\frac{\alpha}{\beta}\right), & \alpha<\beta, \\
\frac{\alpha \pi}{2}, & \alpha>\beta .\end{cases}
\end{aligned}
$$

\section{Ohmic case}

For $s=0$ we can write

$$
\mathcal{G}_{\mathrm{rs}}^{(R)}(\Delta)=\frac{1}{\pi \omega_{0}^{2}} \int_{0}^{\infty} \frac{d k}{k} J_{0}(k|\mathbf{r}-\mathbf{s}|)[1-\cos (k v \Delta)] .
$$

Then, Eq. (23) can be obtained by using the integral [31]

$$
\int_{0}^{\infty} \frac{d x}{x} J_{0}(\beta x)[1-\cos (\alpha x)]=\operatorname{arcosh}\left(\frac{\alpha}{\beta}\right) \theta(\alpha-\beta) .
$$

\section{Super-Ohmic case}

For $s=1 / 2$ we can write

$\mathcal{G}_{\mathbf{r s}}^{(R)}(\Delta)=\frac{v}{\pi \omega_{0}^{3}} \int_{0}^{\infty} d k J_{0}(k|\mathbf{r}-\mathbf{s}|)[1-\cos (k v \Delta)]$.

Then, Eq. (25) can be obtained by using the integral [32]

$$
\int_{0}^{\infty} d x J_{0}(\beta x)[1-\cos (\alpha x)]=\frac{1}{\beta}-\frac{\theta(\beta-\alpha)}{\sqrt{\beta^{2}-\alpha^{2}}} .
$$

\section{APPENDIX C: CORRELATOR $\mathcal{G}_{\mathrm{rs}}^{(I)}(\Delta)$}

The correlator in Eq. (18) can be evaluated in the following way. Starting with Eq. (13), we have

$$
\begin{aligned}
{\left[f\left(\mathbf{r}, t_{1}\right), f\left(\mathbf{s}, t_{2}\right)\right]=} & -2 i \frac{\left(v / \omega_{0}\right)^{D+2 s}}{L^{D}} \sum_{\mathbf{k} \neq 0}|\mathbf{k}|^{2 s} \sin [\mathbf{k} \cdot(\mathbf{r}-\mathbf{s}) \\
& \left.+|\mathbf{k}| v\left(t_{1}-t_{2}\right)\right],
\end{aligned}
$$

which allows us to write

$$
\begin{aligned}
& \frac{1}{2}\left\{\left[f\left(\mathbf{r}, t_{1}\right), f\left(\mathbf{s}, t_{2}\right)\right]+\left[f\left(\mathbf{s}, t_{1}\right), f\left(\mathbf{r}, t_{2}\right)\right]\right\} \\
& \quad=-2 i \frac{\left(v / \omega_{0}\right)^{D+2 s}}{L^{D}} \sum_{\mathbf{k} \neq 0}|\mathbf{k}|^{2 s} \cos [\mathbf{k} \cdot(\mathbf{r}-\mathbf{s})] \sin \left[|\mathbf{k}| v\left(t_{1}-t_{2}\right)\right] .
\end{aligned}
$$

Considering $D=2$, we have

$$
\begin{aligned}
& \frac{1}{2}\left\{\left[f\left(\mathbf{r}, t_{1}\right), f\left(\mathbf{s}, t_{2}\right)\right]+\left[f\left(\mathbf{s}, t_{1}\right), f\left(\mathbf{r}, t_{2}\right)\right]\right\} \\
& \quad=-\frac{i}{\pi}\left(\frac{v}{\omega_{0}}\right)^{2+2 s} \int_{0}^{\Lambda} d k k^{2 s+1} J_{0}(k|\mathbf{r}-\mathbf{s}|) \sin \left[k v\left(t_{1}-t_{2}\right)\right] .
\end{aligned}
$$

In Eq. (C3), we introduced an ultraviolet momentum cutoff $\Lambda$. To proceed further, we need to specify $s$. Below, we consider three representative values.

\section{Sub-Ohmic case}

For $s=-1 / 2$, the integral in Eq. (C3) converges. Using the integral [33]

$$
\int_{0}^{\infty} d x J_{0}(\beta x) \sin (\alpha x)=\operatorname{sgn}(\alpha) \frac{\theta(|\alpha|-|\beta|)}{\sqrt{\alpha^{2}-\beta^{2}}},
$$

we have

$$
\begin{aligned}
& \frac{1}{2}\left\{\left[f\left(\mathbf{r}, t_{1}\right), f\left(\mathbf{s}, t_{2}\right)\right]+\left[f\left(\mathbf{s}, t_{1}\right), f\left(\mathbf{r}, t_{2}\right)\right]\right\} \\
& \quad=-\frac{i}{\pi}\left(\frac{v}{\omega_{0}}\right) \operatorname{sgn}\left(t_{1}-t_{2}\right) \frac{\theta\left(v\left|t_{1}-t_{2}\right|-|\mathbf{r}-\mathbf{s}|\right)}{\sqrt{v^{2}\left|t_{1}-t_{2}\right|^{2}-|\mathbf{r}-\mathbf{s}|^{2}}} .
\end{aligned}
$$

Carrying out the time-ordered integration over $t_{1}$ and $t_{2}$, we obtain Eq. (22).

\section{Ohmic case}

For $s=0$, we notice that

$$
\begin{aligned}
& \frac{1}{2}\left\{\left[f\left(\mathbf{r}, t_{1}\right), f\left(\mathbf{s}, t_{2}\right)\right]+\left[f\left(\mathbf{s}, t_{1}\right), f\left(\mathbf{r}, t_{2}\right)\right]\right\} \\
& \quad=-\frac{i}{\pi}\left(\frac{v}{\omega_{0}}\right)^{2} \frac{d}{d t_{2}} \frac{d}{d t_{1}} \int_{0}^{\Lambda} \frac{d k}{k} J_{0}(k|\mathbf{r}-\mathbf{s}|) \sin \left[k v\left(t_{1}-t_{2}\right)\right] .
\end{aligned}
$$

Using the integral [34]

$$
\begin{aligned}
& \int_{0}^{\infty} \frac{d x}{x} J_{0}(\beta x) \sin (\alpha x) \\
& =\operatorname{sgn}(\alpha)\left[\frac{\pi}{2} \theta(|\alpha|-|\beta|)+\arcsin \left(\frac{|\alpha|}{|\beta|}\right) \theta(|\beta|-|\alpha|)\right]
\end{aligned}
$$

we obtain

$$
\begin{aligned}
\frac{1}{2}\{[ & \left.\left.f\left(\mathbf{r}, t_{1}\right), f\left(\mathbf{s}, t_{2}\right)\right]+\left[f\left(\mathbf{s}, t_{1}\right), f\left(\mathbf{r}, t_{2}\right)\right]\right\} \\
= & -\frac{i}{\pi}\left(\frac{v}{\omega_{0}}\right)^{2} \frac{d}{d t_{2}} \frac{d}{d t_{1}}\left[\frac{\pi}{2} \theta\left(\left|t_{1}-t_{2}\right|-|\mathbf{r}-\mathbf{s}|\right)\right. \\
& \left.+\arcsin \left(\frac{\left|t_{1}-t_{2}\right|}{|\mathbf{r}-\mathbf{s}|}\right) \theta\left(|\mathbf{r}-\mathbf{s}|-\left|t_{1}-t_{2}\right|\right)\right] .
\end{aligned}
$$

Carrying out the time-ordered integration in $t_{1}$ and $t_{2}$, we obtain Eq. (24). 


\section{Super-Ohmic case}

Similarly to the Ohmic case, for $s=1 / 2$ we write

$$
\begin{aligned}
\frac{1}{2} & \left\{\left[f\left(\mathbf{r}, t_{1}\right), f\left(\mathbf{s}, t_{2}\right)\right]+\left[f\left(\mathbf{s}, t_{1}\right), f\left(\mathbf{r}, t_{2}\right)\right]\right\} \\
& =-\frac{i}{\pi}\left(\frac{v}{\omega_{0}}\right)^{3} \frac{d}{d t_{2}} \frac{d}{d t_{1}} \int_{0}^{\Lambda} d k J_{0}(k|\mathbf{r}-\mathbf{s}|) \sin \left[k v\left(t_{1}-t_{2}\right)\right] .
\end{aligned}
$$

Using the integral in Eq. (C4), we obtain

$$
\begin{aligned}
& \frac{1}{2}\left\{\left[f\left(\mathbf{r}, t_{1}\right), f\left(\mathbf{s}, t_{2}\right)\right]+\left[f\left(\mathbf{s}, t_{1}\right), f\left(\mathbf{r}, t_{2}\right)\right]\right\} \\
& =-\frac{i}{\pi}\left(\frac{v}{\omega_{0}}\right)^{3} \frac{d}{d t_{2}} \frac{d}{d t_{1}}\left[\frac{\theta\left(\left|t_{1}-t_{2}\right|-|\mathbf{r}-\mathbf{s}|\right)}{\sqrt{v^{2}\left|t_{1}-t_{2}\right|^{2}-|\mathbf{r}-\mathbf{s}|^{2}}}\right] .
\end{aligned}
$$

Carrying out the integration the time-ordered integrations on $t_{1}$ and $t_{2}$, we arrive at Eq. (26).

\section{APPENDIX D: SINGLE-QUBIT FLIPPING PROBABILITY}

We can obtain a compact expression for the single-qubitbath evolution operator in Eq. (58) by following essentially the same steps shown in Appendix A. The only formal difference is that summations over all qubits in the lattice have to be replaced by a term corresponding to a single qubit $j$. Thus, considering Eq. (15), the result is

$$
U_{j}(\Delta)=\chi_{j}: \exp \left[-\frac{i \lambda}{2} F_{\mathbf{r}_{j}}(\Delta) \sigma_{j}^{x}\right]:,
$$

where

$$
\chi_{j}=\exp \left[-\frac{\lambda^{2}}{8} \mathcal{G}_{\mathbf{r}_{j} \mathbf{r}_{j}}^{(R)}(\Delta)\right] .
$$

since $\mathcal{G}_{\mathbf{r}_{j} \mathbf{r}_{j}}^{(I)}(\Delta)=0$.

Consider now the change of basis

$$
\begin{aligned}
& \left|\uparrow_{j}\right\rangle=\frac{1}{\sqrt{2}}\left(\left|\uparrow_{j}\right\rangle_{x}+\left|\downarrow_{j}\right\rangle_{x}\right), \\
& \left|\downarrow_{j}\right\rangle=\frac{1}{\sqrt{2}}\left(\left|\uparrow_{j}\right\rangle_{x}-\left|\downarrow_{j}\right\rangle_{x}\right),
\end{aligned}
$$

which allows one to write

$$
\left\langle\uparrow_{j}\left|U_{j}(\Delta)\right| \downarrow_{j}\right\rangle=\frac{1}{2}\left\{\exp \left[-\frac{i \lambda}{2} F_{\mathbf{r}_{j}}(\Delta)\right]-\exp \left[\frac{i \lambda}{2} F_{\mathbf{r}_{j}}(\Delta)\right]\right\}
$$

since $: e^{-(i \lambda / 2) F_{\mathrm{r}_{j}}(\Delta)}:=\chi_{j}^{-1} e^{-(i \lambda / 2) F_{\mathrm{r}_{j}}(\Delta)}$ [see Eqs. (A12) and (A13)]. We can then write

$$
\begin{aligned}
& \left\langle\uparrow_{j}\left|U_{j}^{\dagger}(\Delta)\right| \downarrow_{j}\right\rangle\left\langle\downarrow_{j}\left|U_{j}(\Delta)\right| \uparrow_{j}\right\rangle \\
& =\frac{1}{4}\left\{2-\exp \left[i \lambda F_{\mathbf{r}_{j}}(\Delta)\right]-\exp \left[-i \lambda F_{\mathbf{r}_{j}}(\Delta)\right]\right\} \\
& =\frac{1}{4}\left\{2-\chi_{j}^{2}: \exp \left[i \lambda F_{\mathbf{r}_{j}}(\Delta)\right]:-\chi_{j}^{2}: \exp \left[-i \lambda F_{\mathbf{r}_{j}}(\Delta)\right]:\right\},
\end{aligned}
$$

which yields Eq. (59) when the expectation value over the bath vacuum is taken. Now we can insert Eq. (D1) into Eq. (57) to obtain Eq. (59).
[1] S. Bravyi and A. Yu. Kitaev, arXiv:quant-ph/9811052.

[2] M. H. Freedman and D. A. Meyer, Found. Comput. Math. 1, 325 (2001).

[3] E. Dennis, A. Kitaev, A. Landahl, and J. Preskill, J. Math. Phys. 43, 4452 (2002).

[4] A. Yu. Kitaev, Ann. Phys. 303, 2 (2003).

[5] D. S. Wang, A. G. Fowler, and L. C. L. Hollenberg, Phys. Rev. A 83, 020302(R) (2011).

[6] R. Raussendorf and J. Harrington, Phys. Rev. Lett. 98, 190504 (2007).

[7] H. Bombin, R. S. Andrist, M. Ohzeki, H. G. Katzgraber, and M. A. Martin-Delgado, Phys. Rev. X 2, 021004 (2012).

[8] A. G. Fowler, M. Mariantoni, J. M. Martinis, and A. N. Cleland, Phys. Rev. A 86, 032324 (2012).

[9] I. Bloch, J. Dalibard, and S. Nascimbene, Nat. Phys. 8, 267 (2012).

[10] R. Blatt and D. Wineland, Nature (London) 453, 1008 (2008).

[11] M. Saffman, T. G. Walker, and K. Mølmer, Rev. Mod. Phys. 82, 2313 (2010).

[12] M. A. Eriksson, M. Friesen, S. N. Coppersmith, R. Joynt, L. J. Klein, K. Slinker, C. Tahan, P. M. Mooney, J. O. Chu, and S. J. Koester, Quantum Inf. Proc. 3, 133 (2004); H. Bluhm et al., Nat. Phys. 7, 109 (2011).

[13] J. Clarke and F. K. Wilhelm, Nature (London) 453, 1031 (2008); D. P. DiVincenzo, Phys. Scr. T137, 014020 (2009).
[14] E. Novais, E. R. Mucciolo, and H. U. Baranger, Phys. Rev. Lett. 98, 040501 (2007).

[15] E. Novais, E. R. Mucciolo, and H. U. Baranger, Phys. Rev. A 78, 012314 (2008).

[16] E. Novais, E. R. Mucciolo, and H. U. Baranger, Phys. Rev. A 82, 020303(R) (2010).

[17] D. Aharonov, A. Kitaev, and J. Preskill, Phys. Rev. Lett. 96, 050504 (2006).

[18] H. K. Ng and J. Preskill, Phys. Rev. A 79, 032318 (2009).

[19] J. Preskill, Quantum Inf. Comput. 13, 0181 (2013).

[20] E. Novais and E. R. Mucciolo, Phys. Rev. Lett. 110, 010502 (2013).

[21] D. Aharonov, Phys. Rev. A 62, 062311 (2000).

[22] G. Duclos-Cianci and D. Poulin, Phys. Rev. Lett. 104, 050504 (2010).

[23] A. G. Fowler, A. C. Whiteside, and L. C. L. Hollenberg, Phys. Rev. Lett. 108, 180501 (2012).

[24] U. Weiss, Quantum Dissipative Systems (World Scientific, Singapore, 2001).

[25] Notice that Eq. (5) in Ref. [20] has a typographical error.

[26] When the syndromes point to an error, one can try to recover the state by flipping back qubits. However, there is no guarantee that the appropriate string of qubits is being flipped. As a result, the recovery operation may induce a logical error.

[27] N. Madras and G. Slade, The Self-Avoiding Walk (Birkhäuser, Boston, 1996). 
[28] M. Suzuki and M. Katori, J. Phys. Soc. Jpn. 55, 1 (1986).

[29] M. Suzuki, M. Katori, and X. Hu, J. Phys. Soc. Jpn. 56, 3092 (1987).

[30] This expression is obtained using integration by parts and Eqs. 6.56.1.14, 6.693.2, and 6.693.1 from Ref. [31].
[31] I. S. Gradshteyn and I. M. Ryzhik, Table of Integrals, Series, and Products (Academic, San Diego, 1980), Eq. 6.696.

[32] Ref. [31], Eqs. 6.511.5 and 6.671.8.

[33] Ref. [31], Eq. 6.761.7.

[34] Ref. [31], Eq. 6.693.7. 